\title{
Design and Performance Evaluation of Two-UNIT YAgI-UDA ARRAY FOR UHF SATELLITE COMMUNICATION
}

\author{
Rupesh Lad ${ }^{1}$, Pritesh Chhajed ${ }^{2}$, Lokeshsingh Bais ${ }^{3}$, Shyam Dahiwal ${ }^{4}$, Sukhada Saoji ${ }^{5}$, \\ Vaibhav Rekhate ${ }^{6}$, Pushkar Chaudhari ${ }^{7}$, Shimoli Shinde ${ }^{8}$, Ketan Chitale ${ }^{9}$, Anjali \\ Mondhe ${ }^{10}$ and Shreyas Kulkarni ${ }^{11}$ \\ College of Engineering Pune, Pune, India
}

\begin{abstract}
Cube satellite missions perform innovative scientific experiments on a low cost developmental platform but have an inherent limitation of size and space. This restricts the total available solar power that can be harnessed and as a result, the radio links operate on stringent power budgets. For improving the available margins for communication in such satellites, it is desirable to improve upon the antenna system performance at the ground station used for the establishment of the links with the satellite. This can be achieved by improving the forward gain, the forward to backward ratio and the directivity of the antenna. This paper describes the electrical simulations and the performance evaluation of the one unit, two unit and four unit circularly polarized crossed Yagi-Uda antenna array designed for communication with amateur radio (HAM) satellites operating over the $434 \mathrm{MHz}$ to $438 \mathrm{MHz}$ Amateur UHF band. The electro-magnetic model has been developed using the 4NEC2 software. The simulations have been validated with the practical field testing performed for estimating the SWR, antenna gain, the forward to backward ratio and radiation pattern for the antenna system.
\end{abstract}

\section{KEYWORDS}

Array Antenna, polarization, radiation pattern, stacking distance, UHF antenna, Yagi-Uda

\section{INTRODUCTION}

Amateur cube satellites orbiting in Low Earth Orbit are categorized as pico satellites, nano satellites based upon their size. Smaller the size of these cubesats, lesser is the power generation capacity and thus lesser is the power of telemetry signals transmitted from such satellites. Communication with such small cube satellites requires the establishment of an efficient ground system. The performance of the system can be greatly increased by developing a high gain, directional antenna. A specific gain is associated with each type of antenna based upon its structure. A half wave dipole antenna has a nominal gain of about $2.14 \mathrm{dBi}$. It is omnidirectional antenna and thus it has low directivity. When the reflectors and directors are added to the dipole antenna, its gain starts increasing with each director. But the gain gets saturated at about $15 \mathrm{dBi}$, with number of directors equal to 11 [1]. For further increase in the gain, a number of antennas can be coupled together using proper impedance matching network. Horn antennas and parabolic dish antennas have very high directionality but there lie structural constraints and incompatibility with the antenna rotator system. 


\section{Antenna Design}

\subsection{Polarization of ground station antenna}

Elliptical polarization is the most general form of polarization. The loss due to mismatch between polarization of transmitting and receiving antenna is given by

$$
\text { Loss }=10 \log _{10}\left\{\frac{1}{2}+\frac{1}{2}\left[\frac{4 \gamma_{T} \gamma_{R}+\left(1-\gamma_{\mathrm{T}}^{2}\right)\left(1-\gamma_{\mathrm{R}}^{2}\right)(\cos 2 \beta)}{\left(1+\gamma_{\mathrm{T}}^{2}\right)\left(1+\gamma_{\mathrm{R}}^{2}\right)}\right]\right\}
$$

Where, $\gamma_{T}$ is Axial ratio of transmitting antenna, $\gamma_{R}$ is Axial ratio of receiving antenna and $\beta$ is polarization mismatch angle [2].

Small satellites are equipped with linearly polarized dipole antenna transmitting linearly polarized electro-magnetic waves [3]. This linearly polarized wave undergo Faradays rotation as it travels across the space, hence the state of polarization received on the Earth cannot be predicted. Theoretically the polarization loss between the two linearly polarized antennas varies from 0dB to infinite loss depending upon the angle of mismatch given by

$$
\operatorname{Loss}(\mathrm{dB})=10 \log _{10}\left\{\frac{1}{2}+\frac{1}{2}\left[\frac{(\cos 2 \beta)}{1}\right]\right\}
$$

The maximum loss between linear and circularly polarized antenna obtained after substituting values in (1) is $3 \mathrm{~dB}$ [2]. A crossed Yagi antenna reduces the losses due to polarization mismatch.

\subsection{Gain of Antenna}

The satellite downlink power budgets are very stringent and operates with link margin of about $2 \mathrm{~dB}$. Thus to have a large link margin the gain of antenna needs to be more than $15 \mathrm{dBi}$. To receive and extract information from the weak signals arriving at the Earth from satellites, the power of signal must exceed the sensitivity of the receiver and the signal to noise ratio (SNR) needs to be sufficiently higher. Both the objectives of achieving power and SNR can be fulfilled by amplifying the received signals using an antenna and low noise amplifier. High gain antennas also have very high directionality thus the antenna rotator system was needed to direct the main lobe of radiation pattern towards the satellite. The Yeasu $\mathrm{G} 5500^{\mathrm{TM}}$ antenna rotor assembly [4] is mounted on the Antenna mast which elevates the antenna by 2 meters from the roof top.

\subsection{Antenna modeling in $4 \mathrm{NEC2}$}

Simulations for various parameters and dimensions of antenna is carried out in 4NEC2X software which works on Numerical Electromagnetic Codes [5]. It uses method of moments to find out numerical solutions to the integral equation of induced current in metallic structure.

A circularly polarized cross Yagi [6] was simulated to achieve maximum gain at frequency of $437.025 \mathrm{MHz}$ and the corresponding wavelength is $0.6864 \mathrm{~m}$. The resonating length of a dipole antenna is half of wavelength which is $0.3432 \mathrm{~m}$. Yagi antennas are derived from half wave dipole antennas [7] with reflector and directors added to increase directivity and gain in a specific 
direction. The length of reflector is greater than driven element and the lengths of successive directors go on decreasing. The cross Yagi as shown in Fig. 1 are further derived from the Yagi antennas

Boom is the important part of antenna support assembly but it is also an unintended radiating part of antenna. It is generally preferred to electrically insulate the elements and the conducting boom. Presence of conducting boom close to the elements of antenna shortens the electrical length of antenna [8]. Due to this the bandwidth of antenna shifts to higher frequencies. Thus the physical lengths of elements are added with boom correction length which makes it slightly larger than the simulated lengths. Boom correction help to improve the performance of antenna with respect to standing wave ratio (SWR) and gain on the frequency band for which it is designed. The dimensions of the cross Yagi elements are as mentioned in Table I.

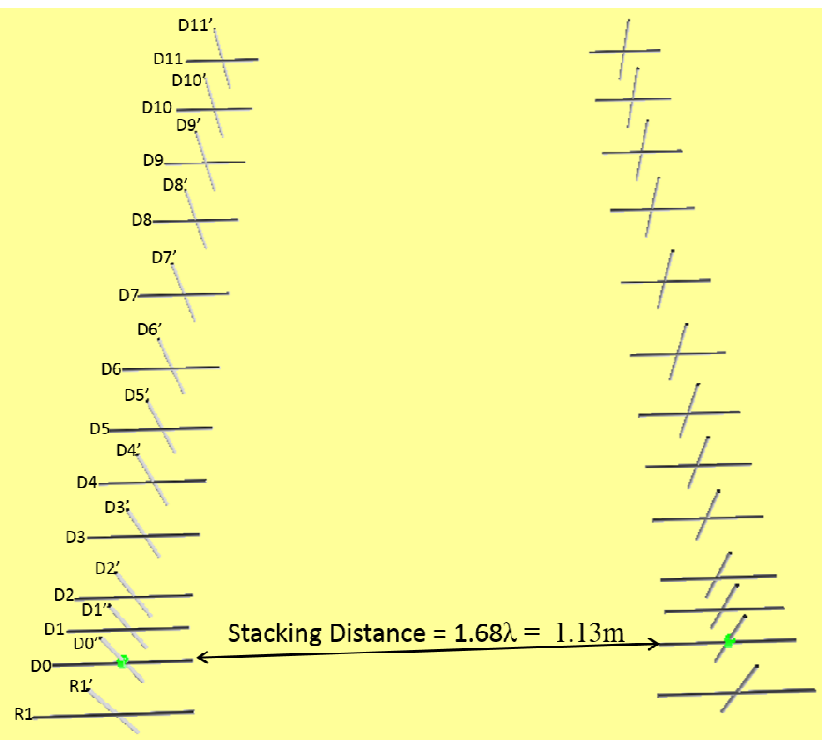

Figure 1. A 2 unit array of Cross Yagi Antenna with elements labelled

The circularly polarized cross Yagi antenna simulated with the above dimensions have a gain of about $15.5 \mathrm{dBi}$ and HPBW of 320. The gain could be further increased by using multiple such antenna and coupling those together [9].

Table 1. Dimensions of antenna elements.

\begin{tabular}{|l|l|l|}
\hline Elements & $\begin{array}{l}\text { Length } \\
\text { (in metres) }\end{array}$ & $\begin{array}{l}\text { Spacing from driven element } \\
\text { (in metres) }\end{array}$ \\
\hline Reflector R1, R1' & 0.3892 & -0.126 \\
\hline Driven elements D0, D0' & 0.3492 & 0 \\
\hline D1, D1' & 0.310 & 0.085 \\
\hline D2, D2' & 0.306 & 0.171 \\
\hline D3, D3' & 0.3022 & 0.342 \\
\hline
\end{tabular}


International Journal of Wireless \& Mobile Networks (IJWMN) Vol. 6, No. 5, October 2014

\begin{tabular}{|l|l|l|}
\hline D4, D4' & 0.2988 & 0.504 \\
\hline D5, D5' & 0.2954 & 0.674 \\
\hline D6, D6' & 0.2918 & 0.884 \\
\hline D7, D7' & 0.2885 & 1.164 \\
\hline D8, D8' & 0.285 & 1.474 \\
\hline D9, D9' & 0.2816 & 1.744 \\
\hline D10, D10' & 0.278 & 2.014 \\
\hline D11, D11' & 0.2744 & 2.284 \\
\hline
\end{tabular}

An array of units of antennas has a narrower beam width, and hence higher gain than one single antenna. The maximum achievable gain could be $\mathrm{N}$ times greater than one unit fed with same power $\left(10 \log _{10} N d B\right)$ if there are $\mathrm{N}$ units in an array. Stacking distance is a function of half power beam width (HPBW) of individual antenna of an array. The optimum stacking distance for maximum gain is given by,

$$
\mathrm{S}_{\mathrm{opt}}=\frac{\lambda}{\left[2 \sin \left(\frac{\phi}{2}\right)\right]}
$$

Where $\phi$ is half power beam width of individual antenna unit [1]. The signals from antennas are combined together and fed to low noise amplifier for further amplification.

The effective gain of an antenna array depends upon the stacking distance between the units of antenna. The optimum stacking distance for 2 unit array antenna obtained from (3) is $1.8 \lambda$. The stacking distance was varied from $0.5 \lambda$ to $3 \lambda$ and the graph of gain vs stacking distance is shown in Fig. 2. It was observed that the gain is maximum at stacking distance of $1.68 \lambda$ and on further increase in stacking distance no significant change in gain is observed. Hence the stacking distance of $1.68 \lambda$ i.e. $1.13 \mathrm{~m}$ is optimum for the present configuration.

Simulations were carried out for 2 unit and 4 unit array using 4NEC2 software and the results are summarized in Table II. Although the primary objective was to build a High gain antenna the losses due to coaxial cables and connectors also have to be considered. The gain obtained from 4 unit cross Yagi antenna is increased but at a cost of physical stability of structure.

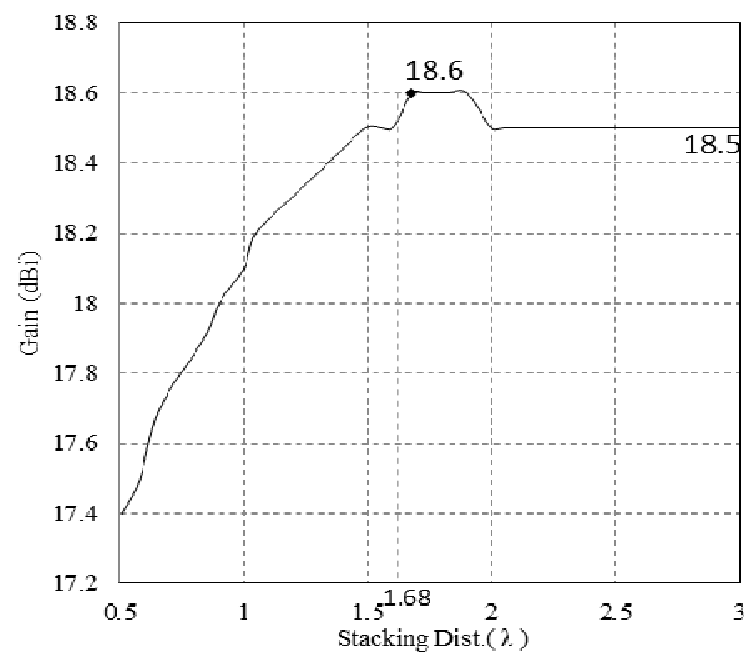

Figure 2. Gain as a function of Stacking distance for 2 unit array antenna 
Table 2. Comparison between antennas

\begin{tabular}{|l|l|l|l|}
\hline Parameter & Cross Yagi antenna & 2 unit array antenna & 4 unit array antenna \\
\hline Gain & $15.5 \mathrm{dBi}$ & $18.6 \mathrm{dBi}$ & $20.9 \mathrm{dBi}$ \\
\hline SWR & 2.8 & 2.78 & 1.49 \\
\hline HPBW & $32^{\circ}$ & $32^{\circ}$ & $16^{\circ}$ \\
\hline Front to Back ratio & 21.35 & 21.86 & 18.36 \\
\hline Cable loss & $0.5 \mathrm{~dB}$ & $1 \mathrm{~dB}$ & $2 \mathrm{~dB}$ \\
\hline Connector loss & $0.22 \mathrm{~dB}$ & $0.44 \mathrm{~dB}$ & $0.88 \mathrm{~dB}$ \\
\hline
\end{tabular}

It is observed that 2 unit array antenna provides higher gain than single cross Yagi antenna and the losses are lesser than 4 unit array antenna. The simulated radiation patterns of 2 unit array antenna are as shown in Fig. 3 and Fig. 4

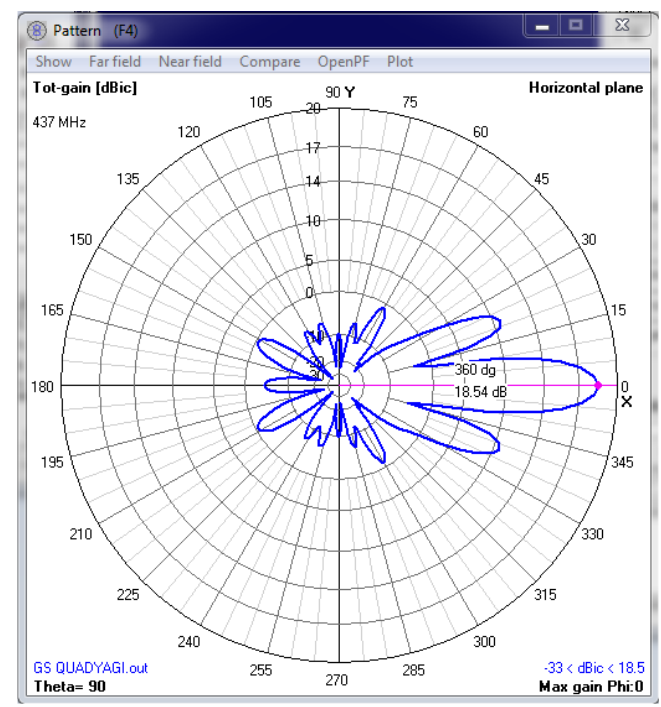

Figure 3. Simulated Horizontal Plane Radiation Pattern 
International Journal of Wireless \& Mobile Networks (IJWMN) Vol. 6, No. 5, October 2014

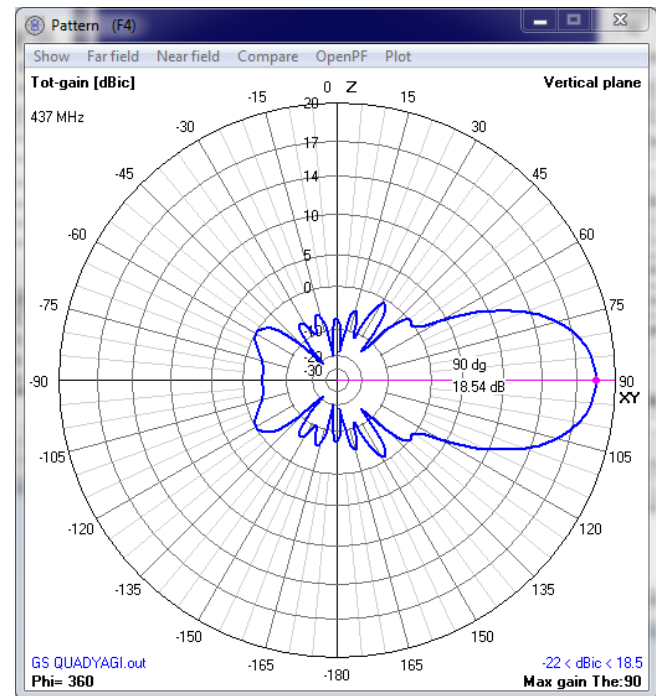

Figure 4. Simulated Vertical Plane Radiation Pattern

\section{FIELd TEST}

The validation of these simulated results is important and is obtained by carrying out various field tests. The 2 unit array antenna is constructed with the simulated dimensions and was mounted on antenna rotor system. A transmitter kept in line of sight and at same height as that of antenna, transmitted a fixed amount of power which was received by Antenna assembly and was measured on Spectrum Analyzer [10]. The antenna was then rotated in horizontal plane and also for different elevation angle. The power received was recorded for each angle and radiation patterns were obtained practically.

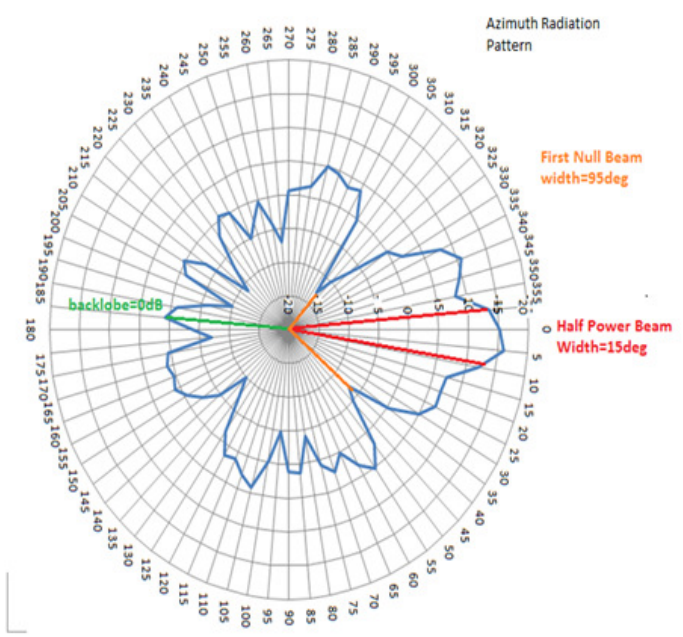

Figure 5. Observed Horizontal Plane Radiation Pattern 


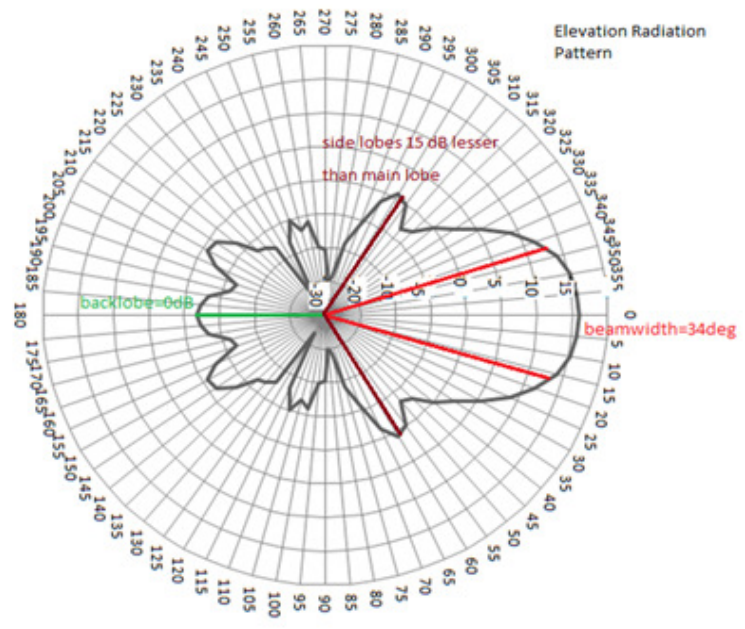

Figure 6. Observed Vertical Plane Radiation Pattern

Presence of metallic bodies in reactive field [11] of antenna contributes to its output parameters and radiation pattern. The physical construction of antenna is such that it avoids use of unintended radiating part in its reactive field region. Use of metallic structure to mount 2 individual units of antenna would have introduced an unintended metallic element hence PVC pipe was used to mount the 2 units on Rotor system thus the practically obtained results are very close to the ones simulated.

The SWR of an antenna can be lowered using matching network [12]-[14] once the antenna is physically constructed hence the gain was given more importance while simulating the antenna. The impedance matching was done using stubs of coaxial cables and with help of smith chart calculations. The SWR of antenna at $437.025 \mathrm{MHz}$ was 2.78 in simulations but the SWR after impedance matching is 1.13 as observed using Vector Network Analyzer [15]. The SWR is lesser than 1.2 for amateur radio satellites frequency range of $434 \mathrm{MHz}$ to $438 \mathrm{MHz}$ with a gain of 17 dBi.

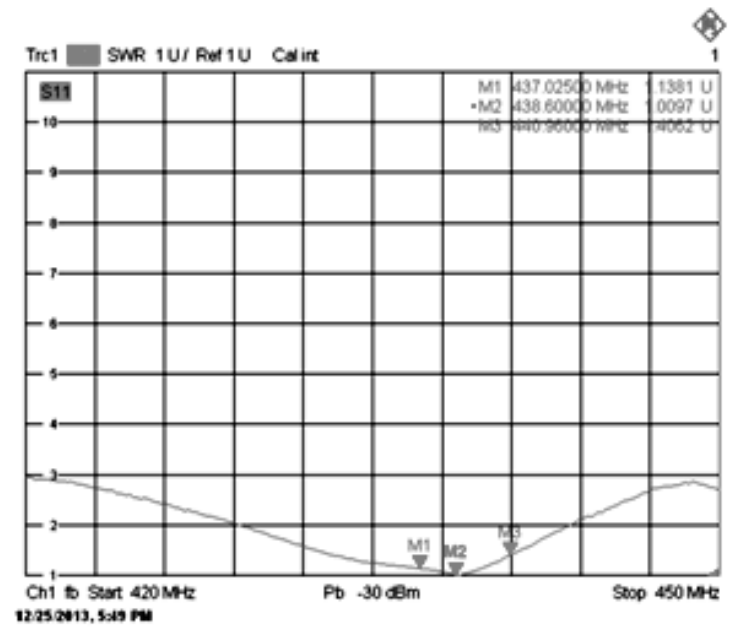

Figure 7. Observed SWR plot as a function of Frequency 


\section{CONCLusion}

The patterns obtained and the field tests are in good congruence with the model developed. Large number of iterative simulations carried out resulted into a comparison of antennas and their performance parameters. Mutual coupling and matching network lowered down the SWR which was high in simulations resulting into high gain and matched antenna. Successful communication links have been practically established with existing amateur radio satellites thus establishing the performance of this antenna.

\section{ACKNOWLEDGEMENTS}

This work is sponsored by College of Engineering, Pune under the Swayam Satellite project. The authors acknowledge the valuable inputs of faculty of Electronics and Telecommunication department and HAM club VU2COE. Authors thank Shri. Pramod Kale for his deep insights in radio and satellite communication. Authors thank Dr. A. D. Sahasrabudhe, Hon. Director of COEP and Dr. M. Y. Khaladkar for their constant motivation and support to the Swayam project.

\section{REFERENCES.}

[1] P. Swallow, "Practical VHF/UHF antennas", in Radio communication handbook, 11th ed. London, UK: The Radio Society of Great Britian, ch. 16.

[2] T. K. Ishii, Handbook of Microwave Technology, Hill and Smith, Volume 2, ch. 7, pp. 176-177.

[3] P. Chaudhari et al., "Performance optimization of a 1-U satellite antenna", in Proc. of 64th International Astronautical Congress, Beijing, China, Sept 2013.

[4] Yeasu G5500 Antenna Azimuth Elevation Rotator and Controller Operating Manual, Yeasu Musen Co., Ltd., 1998.

[5] Numerical electomagnetic code (NEC-1),"NEC User's guide", Lawrence Livermore Laboratory, 1977.

[6] C. A. Balanis, Antenna Theory Analysis and Design, John Willey\& Sons, inc, New York, Chichecter, Brisbane, Toronto, Singapore, 2003

[7] R. K. Shevgaonkar, "Antennas”, in Electromagnetic Waves, New Delhi, India: Tata McGraw-Hill, 2006, ch. 8.

[8] D. Dragoslav, "Boom distance influence on yagi antenna", in Antennex, Issue 148, Serbia, August 2009.

[9] M. Pingle, B. Pingle, S. Deosarkar, "Mutual coupling in arrays and its effect on $435 \mathrm{MHz} 4-$ element Yagi-Uda antennas in array configurations", in Proc. of International Conference on Recent Advances in Microwave Theory and Applications, Jaipur, Nov 2008; pp. 818-820.

[10] R\&S FSH Handheld Spectrum Analyzers Operating Manual, Rohde \& Schwarz, pp. 2.20-2.23.

[11] A. W. Rudge, Handbook of Antenna Design, Volume 1, pp. 12-14.

[12] D. Jefferies, Single stub matching of transmission lines to loads, [Online] Available: http://personal.ee.surrey.ac.uk/Personal/D.Jefferies/jefferies-stub.html

[13] J. Staples, Impedance matching and smith charts, Available: http://uspas.fnal.gov/materials/08UCSC/mml13_matching+smith_chart.pdf

[14] R. K. Shevgaonkar, "Transmission Lines", in Electromagnetic Waves, New Delhi, India: Tata McGraw-Hill, 2006, ch. 2.

[15] Performing Amplifier Measurements with the Vector Network Analyzer ZVB, Rohde \& Schwarz, pp. 3-7. 